\title{
Stability Studies of Torque Transducers
}

\author{
Anil Kumar, Harish Kumar \\ National Physical Laboratory (Council of Scientific \& Industrial Research), New Delhi, 110012, India \\ E-mail: ak_npl@rediffmail.com
}

The present paper discusses the stability studies carried out on a small number of torque transducers over the years with capacities ranging from $10 \mathrm{Nm}$ to $1000 \mathrm{Nm}$. The torque transducers have been calibrated using the torque standard machine based on the written standard calibration procedure. The study reveals that the uncertainty of measurement of torque transducers has been varying appreciably and it is more for lower range. Besides, the deviation from their average values has also been studied and found to be varying through the years.

Keywords: torque transducer, torque standard machine, stability

\section{INTRODUCTION}

$\mathrm{T}$ ORQUE MEASUREMENT plays a vital role in various fields of science and requires a precision measurement method. A torque transducer may be judged on the basis of its metrological performance which may be summarized in terms of the uncertainty of measurement. The uncertainty of measurement is derived from comparing the torque transducer under test to the torque standard machine with known uncertainty and has traceability to the national standards. As per the standard BS 7882 guidelines, when any torque transducer has been calibrated, it is mandatory to indicate the quality of results in terms of repeatability error, reproducibility error, zero error, resolution error, uncertainty of measurement, etc. The present paper discusses the stability studies of a number of torque transducers with capacities ranging from $10 \mathrm{Nm}$ to $1000 \mathrm{Nm}$ calibrated at the National Physical Laboratory, India (NPLI). For the present study, torque standard machine of capacity $2000 \mathrm{Nm}$ has been used. The bmc is determined as $\pm 0.05 \%(k=2)$ for torque standard machines. The torque proving instruments are calibrated as per the standard calibration procedure based on standard BS 7882. The uncertainty of measurement of the torque transducers under study involves the deviations due to zero error, repeatability error, reproducibility error, resolution error and the uncertainty of measurement of torque due to the torque standard machine [1-5].

\section{EXPERIMENTAL SETUP AND CALIBRATION PROCEDURE}

Different types of torque measuring devices are widely used for varying capacities depending upon their applications like power measurement of engine, turbine or other rotating machines in addition to precision measurement of torque, but strain gauged torque transducers are used widely and found to have the precise measurement of torque. These torque transducers consist of a sensing element with strain gauges implanted at suitable locations to measure the strain when the torque transducer is under external torque. The suitable data acquisition system enables to get the electrical output in form of $\mathrm{mV}$, Nm or any other form (Fig.1).
For the present study, torque standard machine of capacity $2000 \mathrm{Nm}$ has been used. Calibration of torque transducers is accomplished by loading the torque transducer into the torque standard machine to the beam of length $1 \mathrm{~m}$ on either side of the torque transducer. One side of the beam has balancing weight and the other side has a hanger so that calibrated weight of known value in terms of newtons, e.g., $20 \mathrm{~N}, 50 \mathrm{~N}$ etc., may be applied depending upon the capacity of torque transducer and the torque required to be generated. The torque standard machine has been compared with the primary torque standard machine (bmc $\pm 0.01 \%$ $(k=2))$ by means of precision torque transfer standards.
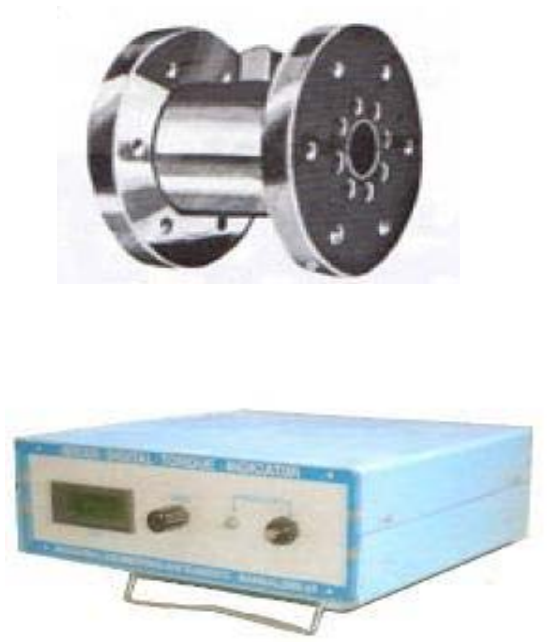

Fig.1. A typical torque transducers with digital indicator

These torque transfer standards are precision strain gauge type having the uncertainty $\pm 0.025 \%$. The torque transfer standards are calibrated directly against the national torque standards over whole of the range as per the standard calibration procedure based on standard BS 7882. The bmc of the torque standard machine of capacity $2000 \mathrm{Nm}$ has been found to be $\pm 0.05 \%(k=2)$. The environmental conditions have been maintained (temperature $23 \pm 1^{\mathrm{O}} \mathrm{C}$, relative humidity $50 \pm 10 \%$ ). 
- The calibration of the torque transducer was carried out by applying a series of torque calibrations in increasing order from $10 \%$ to $100 \%$ in incremental steps of $10 \%$. Two series were taken at $0^{\circ}$. The torque transducer has been rotated to $90^{\circ}$ and $180^{\circ} / 270^{\circ}$ and taken one series of increasing torque calibrations .

- If is not possible to rotate the torque transducer, the torque transducer may be removed from the torque standard machine and may be remounted to the torque standard machine to take remaining series of torque calibrations.

- The uncertainty of measurement of the torque transducer involves the deviations due to zero error, repeatability error, reproducibility error, resolution error and the uncertainty of measurement of torque due to the torque standard machine. The uncertainty of measurement has been computed based on guidelines as per standard [1-5].

- Deviation from average value is given as Deviation $(\%)=100 *\left(X^{\prime \prime}-X^{\prime}\right) / X^{\prime \prime}$

where, $\mathrm{X}^{\prime}$ represent the average value for the given torque in a respective year, e.g., 2006 or 2008 etc., while X"

represents the average value of torque for all years, e.g., 2006, 2008 and 2010.

\section{RESULTS AND DISCUSSION}

National Physical Laboratory, India (NPLI) is the custodian of national standards in India and its charter is to provide the traceability of national standards of torque to the user industries or calibration laboratories. Force and Hardness standard group at NPLI receives a number of torque transducers and has acquired a calibration data bank over the years because of regularly received instruments.

The study has been conducted with a number of torque transducers of varying capacities, but it may not be possible to discuss all of them in detail. Hence, three torque transducers of capacities $10 \mathrm{Nm}$ (clockwise direction, calibrated during 2007, 2008 \& 2009), 100 Nm (clockwise direction, calibrated during 2006, 2008 \& 2010) and 1000 Nm (clockwise direction, calibrated during 2006, 2008 \& 2010) are discussed. The torque transducers selected may have up to $\pm 0.5 \%$ variations in their values as specified by the manufacturer. Their previous data has been tabulated and studied for their uncertainty of torque measurement and deviation w.r.t. the average of the three times calibration results (Fig.2-13).

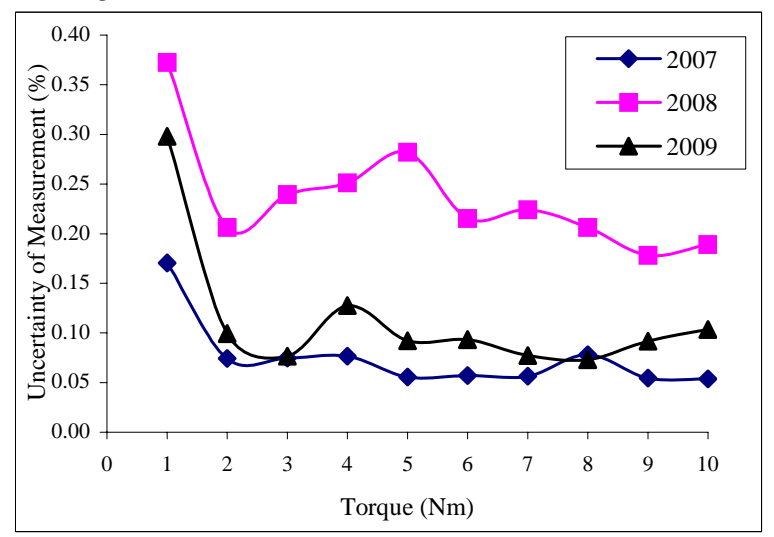

Fig.2. Uncertainty of measurement of $10 \mathrm{Nm}$ transducer
The torque transducer of $10 \mathrm{Nm}$ suggests that the repeatability error and reproducibility error were maximum in 2008 and were the main contributory factor for the uncertainty of measurement. Hence, the uncertainty of measurement of the torque transfer is highest in 2008. The deviation from the average value is maximum in 2009. Variations in resolution error and zero error are found to be negligible (Fig.2-5).

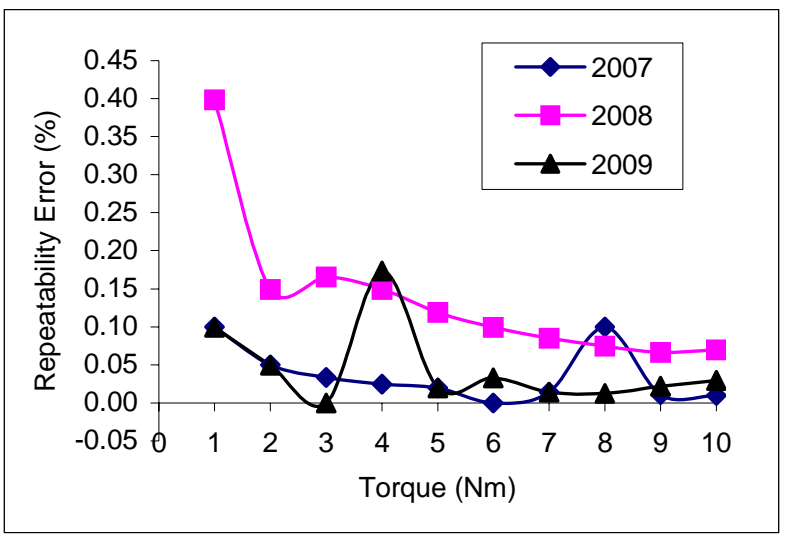

Fig.3. Repeatability error of $10 \mathrm{Nm}$ torque transducer

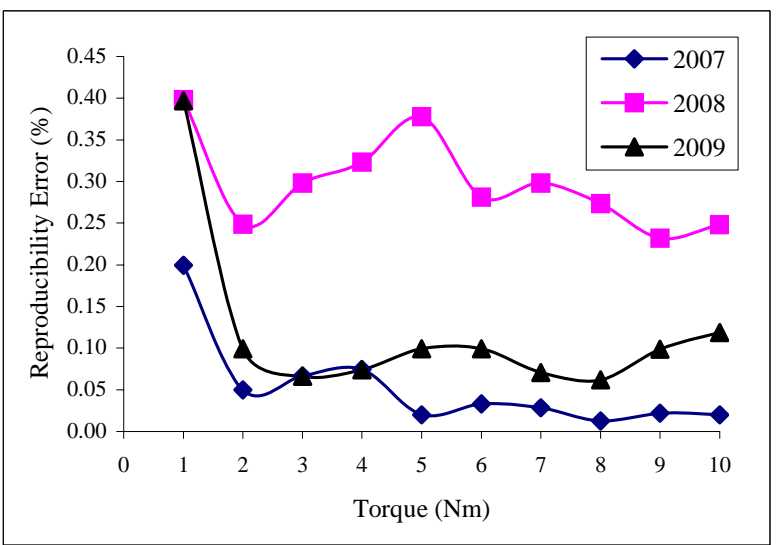

Fig.4. Reproducibility error of $10 \mathrm{Nm}$ torque transducer

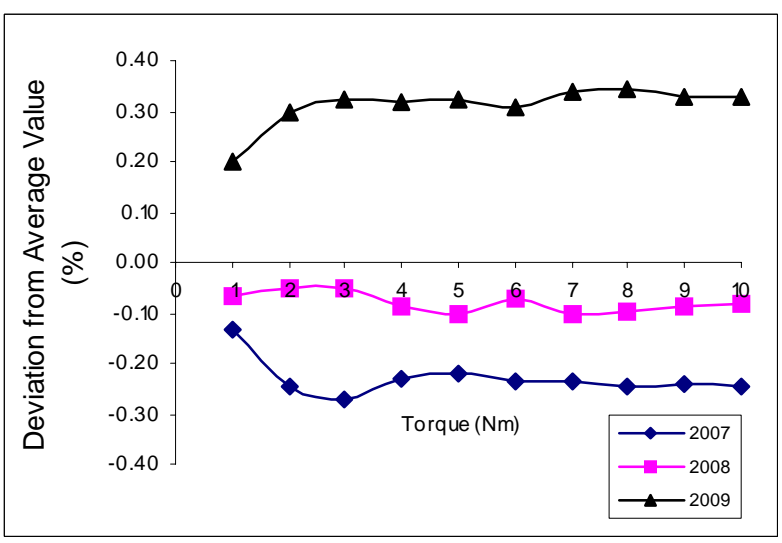

Fig.5. Deviation from average value for $10 \mathrm{Nm}$ torque transducer 
The torque transducer of $100 \mathrm{Nm}$ suggests that the uncertainty of measurement, repeatability error and reproducibility error are were higher in 2006 and 2010. Also, the deviation from the mean value is was higher in 2006 and 2010 and it is was higher is in the lower range of the torque transducer. Resolution error and zero error are very less small and their variation is also insignificant, hence not shown (Fig.6-9).

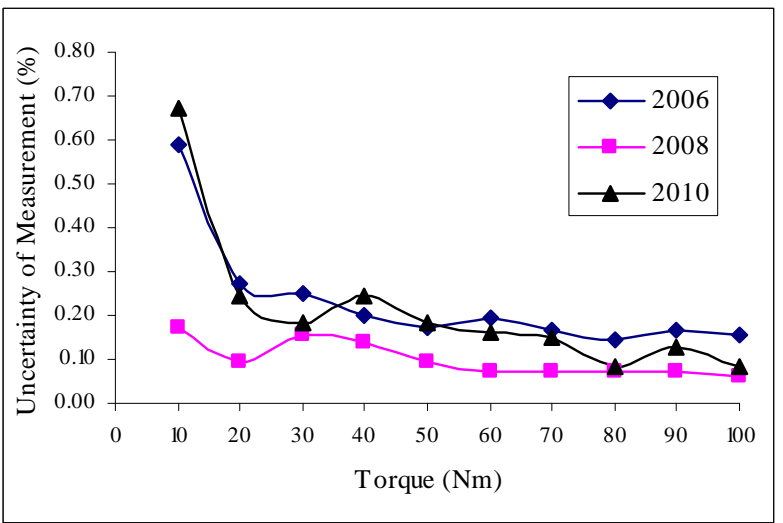

Fig.6. Uncertainty of measurement of $100 \mathrm{Nm}$ torque transducer

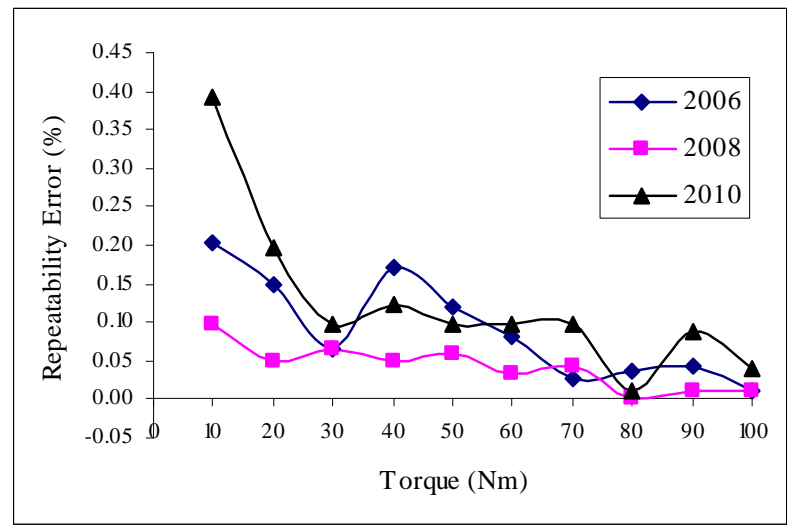

Fig.7. Repeatability error of $100 \mathrm{Nm}$ torque transducer

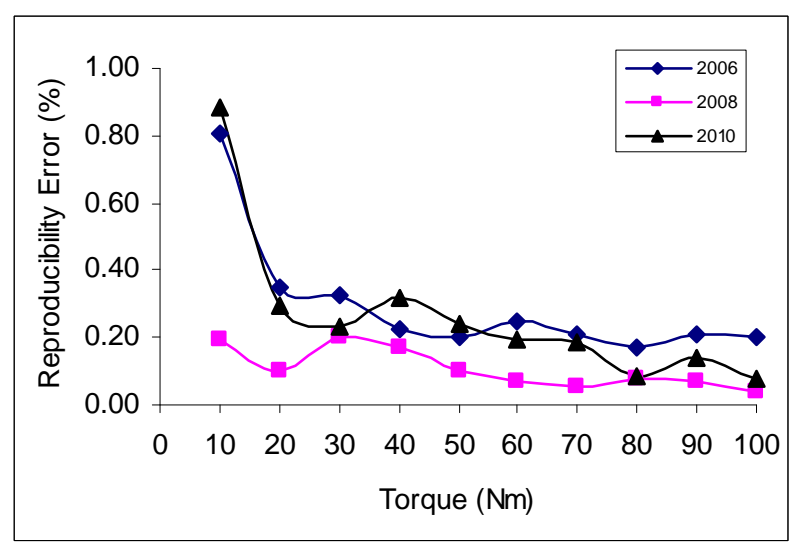

Fig.8. Reproducibility error of $100 \mathrm{Nm}$ torque transducer

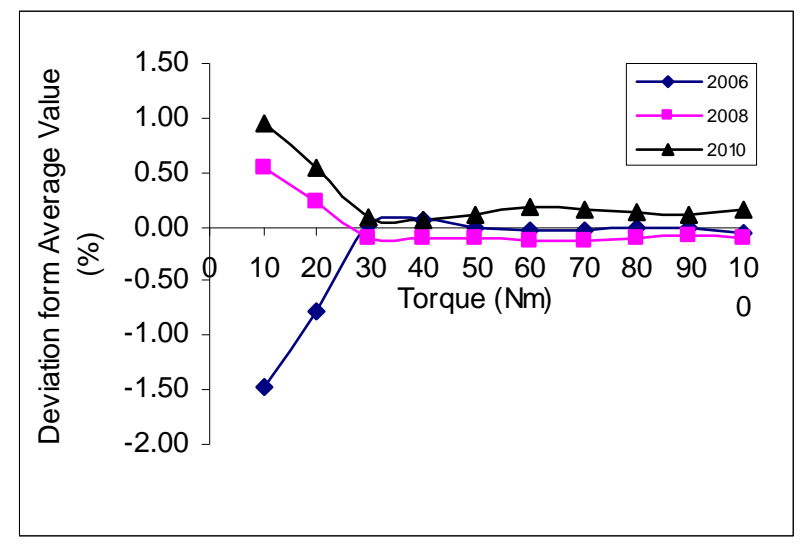

Fig.9. Deviation from average value for $100 \mathrm{Nm}$ torque transducer

The study of torque transducer of $1000 \mathrm{Nm}$ indicates that uncertainty of measurement, repeatability error and reproducibility error has had very less little variation in the respective years. But, the deviation form from the average value is very less low in 2008 in comparison to 2006 and 2010 (Fig.10-13).

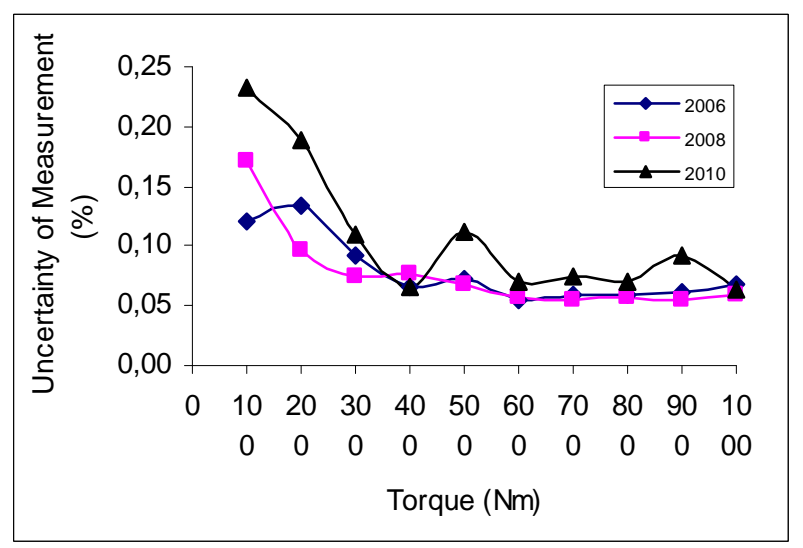

Fig.10. Uncertainty of measurement of $1000 \mathrm{Nm}$ torque transducer

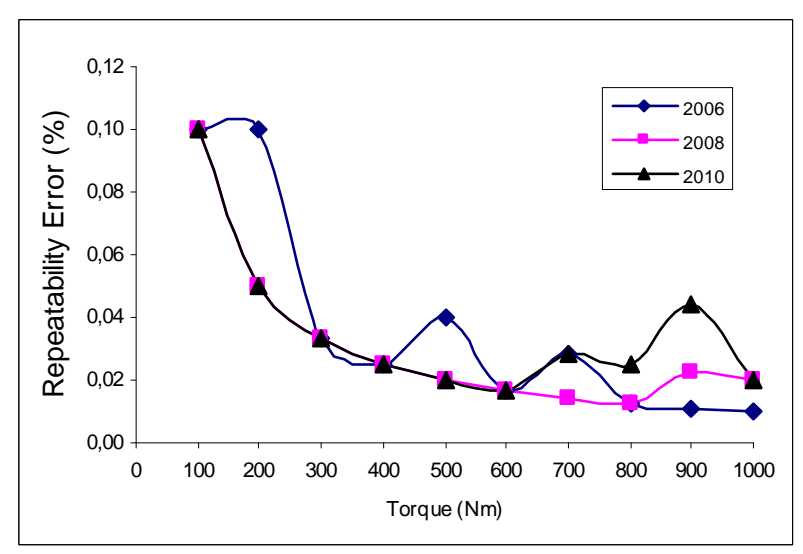

Fig.11. Repeatability error of $100 \mathrm{Nm}$ torque transducer 


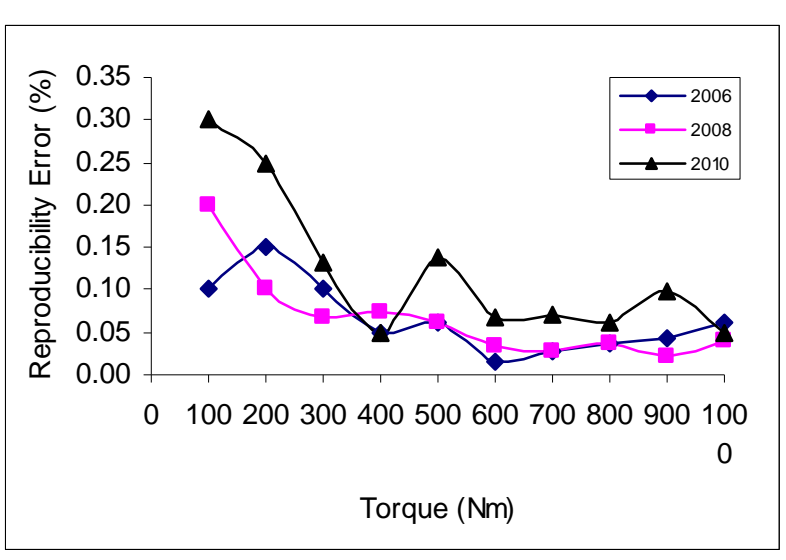

Fig.12. Reproducibility error of $1000 \mathrm{Nm}$ torque transducer

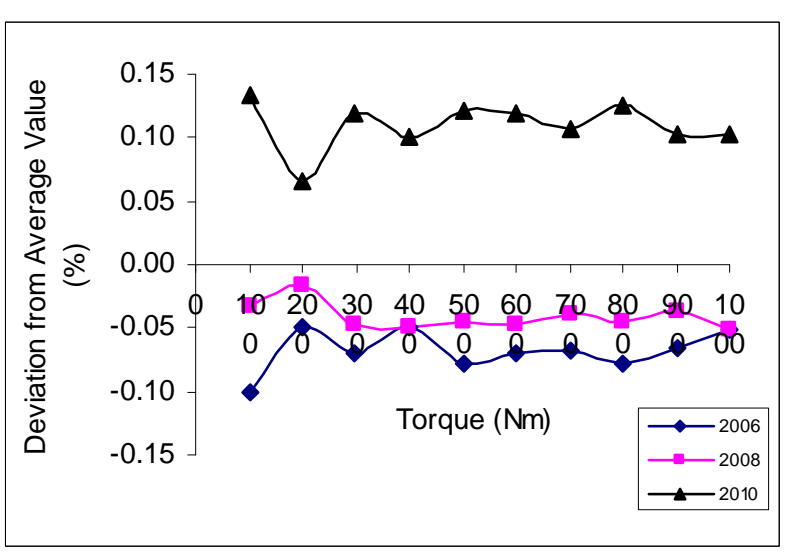

Fig.13. Deviation from average value for $1000 \mathrm{Nm}$ torque transducer

\section{CONCLUSIONS}

Some torque transducers have been studied for their stability over the years using their calibration values against the torque standard machines in the range of $10 \mathrm{Nm}$ to 1000 Nm. The stability study suggests that the values are varyingvary with time and the variation is high in the lower range of the torque transducers. The higher variations in the lower range of torque transducers may be attributed due to poor resolution and higher relative deviations due to repeatability error and reproducibility error. The repeatability error, reproducibility error and deviation from average value have been varying significantly through the years for the torque transducers over the years. The variations do not represent any trend in a particular direction and may be random in nature. Hence, the study provides a scope for a strict vigil over the behavior of torque transducers over the years and if values vary beyond the manufacturer's specifications, an intensive analysis need is necessary to be done for deciding the suitability of the torque transducer.

\section{REFERENCES}

[1] British Standards (2008). Method for Calibration and Classification of Torque Measuring Devices. BS 78822008.

[2] International Standards Organization (1995). ISO GUM Document - Guide for Estimation and Expression of Uncertainty of Measurement.

[3] European Association for Accreditation of Laboratories (1997). Expression of Uncertainty of Measurement in Calibration.

[4] National Accreditation Board for Testing and Calibration Laboratories, India (2000). NABL 141 Guidelines for Estimation and Expression of Uncertainty in Measurement.

[5] Force \& Hardness Standard, National Physical Laboratory, India. Document Manual Group. NPLI_D 5.05/ Doc. 3/ CP \# T-01.

Received March 21, 2011. Accepted May 30, 2011. 